\section{Studie: Wie Menschen mit Rheuma die Corona-Krise erleben}

Die Folgen der Corona-Pandemie erleben Menschen sehr unterschiedlich. Je nach Lebenssituation stehen wirtschaftliche oder auch soziale Auswirkungen im Vordergrund. Ganz besonders belastet sind sicherlich die Menschen, die das Robert Koch-Institut (RKI) zur Risikogruppe für einen schweren Krankheitsverlauf zählt, darunter Menschen mit aktiven entzündlich-rheumatischen Erkrankungen und Patienten unter immunsuppressiver Therapie.

Wie diese Betroffenen mit der Information über ein erhöhtes Risiko umgehen, welche Sorgen und Ängste damit verbunden sind und wie sich die aktuelle Situation auf die rheumatologische Versorgung sowie die Grunderkrankung auswirkt, möchte die Deutsche Gesellschaft für Rheumatologie e.V. (DGRh) nun mithilfe einer Patientenbefragung klären.

Rund $2 \%$ der erwachsenen Bevölkerung Deutschlands sind von entzündlich-rheumatischen Erkrankungen betroffen. Noch lässt die derzeitige Datenlage zwar keine gesicherten Schlüsse darüber zu, wie sich eine solche Vorerkrankung auf den Verlauf einer SARS-CoV-2-Infektion auswirkt. Denn zum einen bindet und erschöpft das entzündliche Geschehen die Kapazitäten des Immunsystems. Zum anderen nehmen viele Rheumapatienten Medikamente wie Methotrexat, Cortisonpräparate oder Biologika ein, die die Immunabwehr beeinträchtigen können.

Viele Patienten sind deshalb verunsichert. Die aktuelle Patientenbefragung soll dazu beitragen, ihre Situation besser zu verstehen und zu untersuchen, ob die Pandemie sich auf die rheumatologische Versorgung auswirkt: „Beeinflussen die Veränderungen im Rahmen der Coronakrise - angefangen bei möglicher Jobunsicherheit bis hin zu einer veränderten Schlafqualität oder dem Umfang an sportlicher Aktivität - die Versorgung und die Krankheitsaktivität unserer Patienten? Können wir sie mit Telemedizin derzeit adäquat erreichen und versorgen? Wie geht es unseren Patienten damit? - diese Fragen stehen im Fokus der Befragung“, erläutert Prof. Bimba Hoyer von der Universität Kiel, die gemeinsam mit Dr. Rebecca Hasseli sowie der Abteilung für Klinische Psychologie von der Justus-Liebig-Universität Gießen und Dr. Tim Schmeiser vom Krankenhaus St. Josef, Wuppertal zur „Task Force“ Patientenumfrage der DGRh zählen. Fragen zur rheumatischen Erkrankung, zur Medikation und Untersuchungen nehmen einen breiten Raum ein, ebenso Fragen nach der Durchführung von Physiotherapie und Änderungen der Job- und/oder der Lebenssituation (home office, home schooling). Ein weiterer Schwerpunkt liegt schließlich auf möglichen psychischen Auswirkungen der Krise wie Ängsten und Sorgen.

„Für uns ist es auch wichtig zu wissen, ob die Patienten nun etwa dazu tendieren, ihre Medikamente ohne ärztlichen Rat abzusetzen oder zu reduzieren“, greift Prof. Christof Specker, Direktor der Klinik für Rheumatologie \& Klinische Immunologie am Evangelischen Krankenhaus der Kliniken Essen-Mitte und DGRh-Vorstandsmitglied, ein weiteres Beispiel heraus . Das sei auf keinen Fall ratsam. Auch warnt er davor, den Kontakt zum Rheumatologen und anderen Ärzten oder Therapeuten aus Angst vor einer Infektion abzubrechen - auch Art und Umfang der Arztkontakte werden daher abgefragt.

Die Umfrage spricht alle Menschen mit Rheuma an - unabhängig davon, ob sie an Covid-19 erkrankt sind/waren oder nicht. Wer sich als Teilnehmer registriert, wird gebeten, den digitalen Fragebogen über ein Jahr hinweg einmal monatlich auszufüllen. „Wir sind hier auf die Mithilfe der Patienten angewiesen", sagt Prof. Hendrik SchulzeKoops, Präsident der DGRh. „Nur wenn möglichst viele Patienten Rückmeldung zu ihrer Situation geben, können wir grundlegende Probleme erkennen und Versorgungslücken schließen“, betont Prof. Ulf MüllerLadner, wissenschaftlicher Leiter der Initiative Patientenbefragung für den Vorstand der DGRh. 
Die aktuelle Befragung ist eine Ergänzung zum Covid-19-Register, das die DGRh bereits vor einigen Wochen ins Leben gerufen hat. Darin dokumentieren Rheumatologen medizinische Informationen über ihre rheumatologischen Patienten, die sich mit SARSCoV-2 infiziert haben. Anonymisiert werden dort Angaben erfasst, wie Alter, Art der Erkrankung und Medikation sowie der Verlauf der Covid-19-Erkrankung. „Bereits jetzt sind hier Daten von über 150 Patienten erfasst“, sagt Schulze-Koops - ein überaus wertvoller Datensatz. Denn mithilfe der ständig aktualisierten Daten lässt sich womöglich bald genauer abschätzen, welche RheumaPatienten während der SARS-CoV-2-Pandemie besonders gefährdet sind - und wie man dieser Gefahr begegnen kann.

Informationen zum Covid19-Register und erste Auswertungen finden sich unter der Adresse https://www.covid19-rheuma.de. Wer an der Patientenbefragung teilnehmen möchte, kann sich unter https://www.covid19-rheuma.de/patienten-information registrieren. Das Register und der Fragebogen werden von der „Ad hoc Kommission COVID-19-Register der DGRh“ entwickelt und wissenschaftlich ausgewertet. Beide Projekte sind in einer gemeinsamen Initiative der DGRh und der Professur für Innere Medizin mit Schwerpunkt Rheumatologie der Justus-Liebig-Universität Gießen entstanden. Die Daten dieses deutschen Registers sollen unter Wahrung des Datenschutzes soweit möglich auch in das internationale Projekt „COVID-19 Global Rheumatology Alliance“ einfließen, um diesen weltweiten Überblick zu unterstützen und zugleich Verständnis von den Besonderheiten aus den verschiedenen Regionen zu generieren.

Nach einer Pressemitteilung der DGRh 